\title{
La entrevista sobre la escritura con alumnado de Primaria: un espacio para la reflexión metalingüística *
}

\section{The Interview about Writing Processes with Primary School Pupils: A Place for Metalinguistic Reflection}

\section{Carmen Rodríguez-Gonzalo}

Universitat de València-GIEL

crodrig@uv.es

ORCID ID: https://orcid.org/0000-0002-8013-7503

\section{Carme Durán Rivas}

Universitat Autònoma de Barcelona

cduran4@gmail.com

ORCID ID: https://orcid.org/0000-0003-2357-1124

\section{Teresa Ribas Seix}

Universitat Autònoma de Barcelona

teresa.ribas@uab.cat

ORCID ID: https://orcid.org/0000-0001-6358-8014

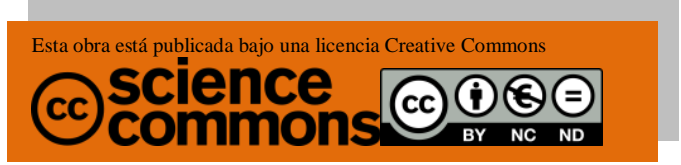

DOI: 10.17398/1988-8430.35.2.103

Fecha de recepción: 26/05/2021

Fecha de aceptación: 26/09/2021

Rodríguez-Gonzalo, C., Durán Rivas, C, y Ribas Seix, T. (2022). La entrevista sobre la escritura con alumnado de Primaria: un espacio para la reflexión metalingüística. Tejuelo, 35(2), 103-136.

Doi: https://doi.org/10.17398/1988-8430.35.2.103

\footnotetext{
* La investigación ha recibido fondos del Ministerio de Economía y Competitividad (proyecto I+D: "Enseñar a escribir: reflexión gramatical y estrategias de autorregulación”-EDU2015-64798-R) así como de ACUP/RecerCaixa con el Proyecto: “Ara l'escriptura! Millorar l'expressió escrita per garantir l'equitat de l'alumnat" (2015ACUP 00175). Se ha concluido en el marco del proyecto I+D "La elaboración de una gramática escolar interlingüística: hacia una enseñanza reflexiva de las lenguas en contextos multilingües (PID2019-105298RB-I00) del Ministerio de Ciencia e Innovación-Agencia Estatal de Investigación.
} 
Resumen: Ante el debate actual sobre la oportunidad y el sentido de enseñar gramática en la escuela, las autoras se adhieren a la tendencia de las últimas décadas de centrar la atención en la actividad metalingüística del aprendiz y de apostar por una enseñanza reflexiva de la gramática. En una investigación con datos empíricos de aula, analizados desde una perspectiva cualitativa, se realizan entrevistas a alumnos de Primaria (7 y 9 años) para profundizar en la actividad metalingüística que manifiestan y para mostrar la entrevista como un espacio que hace emerger la reflexión. Los resultados aportan evidencias de que en Primaria los alumnos pueden verbalizar sus reflexiones sobre la lengua, si bien con algunas condiciones, como situar las tareas en contextos con sentido, disponer de la mediación adecuada del adulto así como compartir metalenguaje para referirse a los fenómenos metalingüísticos. Se desprenden de ahí importantes implicaciones didácticas: hace falta diseñar espacios en el aula para que la reflexión metalingüística de niños y niñas pueda emerger, con la adecuada ayuda docente. Para que ello sea posible, es necesario incorporar en la formación inicial del profesorado criterios sobre la gestión de la interacción para el aprendizaje $\mathrm{y}$, en particular, de las interacciones metalingüísticas.

Palabras clave: reflexión metalingüística; entrevista; escritura; conectores discursivos; Educación Primaria.
Abstract: Given the current debate on the opportunity and meaning of teaching grammar in schools, the authors adhere to the trend of recent decades to focus attention on the metalinguistic activity of the learner and to bet on a reflective teaching of grammar. In a research with empirical classroom data, analyzed from a qualitative perspective, interviews were conducted with Primary School students (7 and 9 years old) to delve into the metalinguistic activity they manifest and to show the interview as a space that makes reflection emerge. The results provide evidence that in Primary Education, students can verbalize their reflections on the language, although with some conditions such as placing tasks in meaningful contexts, having adequate adult mediation as well as sharing metalanguage to refer to metalinguistic phenomena. Important didactic implications emerge from this: it is necessary to design spaces in the classroom so that the metalinguistic reflection of schoolboys and schoolgirls can emerge, with the appropriate teaching help. For this to be possible, it is necessary to incorporate criteria on the management of interaction for learning and, in particular, metalinguistic interactions in the initial teacher training.

Keywords: metalinguistic reflection; interview; writing; discourse connectors; Primary Education. 


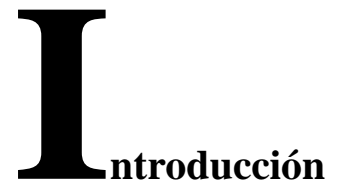

El debate iniciado en los países anglosajones sobre la utilidad de enseñar gramática en la escuela, sobre todo en las primeras edades de la escolaridad obligatoria, sigue vigente en el ámbito internacional (Locke, 2010). No obstante, desde hace unas décadas, un conjunto de trabajos basados en datos empíricos realizados en el área hispánica plantea la necesidad de desplazar la discusión hacia un nuevo foco, el concepto de actividad metalingüística, es decir, la capacidad humana de observar y analizar la lengua y su uso (Camps y Milian, 1999; Camps, 2000; Camps y Fontich, 2020), y de apostar por una enseñanza gramatical organizada mediante la reflexión sobre la lengua en un contexto de diálogo entre iguales y con ayuda del docente.

El trabajo que presentamos corresponde a una investigación sobre enseñanza de la escritura con alumnos de enseñanza obligatoria, centrada en propiciar didácticamente la capacidad de autorregulación de los estudiantes (Allal, 1993; Ribas, 2001) mediante el diálogo generado en clase. En un trabajo reciente (Ribas, Rodríguez-Gonzalo y Durán, 
2020) sobre el proceso de aprendizaje de la escritura en edades tempranas, analizamos las características de la actividad metalingüística de alumnos de $4^{\circ}$ de Primaria, contrastando los datos obtenidos durante el proceso y los datos del producto. En el artículo que ahora presentamos, centramos el análisis en las entrevistas realizadas a alumnos de $2^{\circ}$ y $4^{\circ}$ de Primaria (7 y 9 años respectivamente) durante una Secuencia Didáctica (en adelante, SD) cuyo fin es la escritura de un texto de opinión (Salas, Birello y Ribas, 2020). Las entrevistas permiten acceder a las concepciones del alumnado sobre la escritura y sobre la función de los conectores discursivos y al mismo tiempo crean un contexto donde la reflexión sobre la lengua es posible y donde aparecen trazas de la actividad metalingüística verbalizada de los estudiantes (Camps, 2000; Fontich y García-Folgado, 2018).

En los últimos años se ha defendido el interés por estimular la reflexión sobre la lengua de los estudiantes ya en edades tempranas. Desde el marco sociocultural, se ha puesto el énfasis en los procesos de toma de conciencia y en la reflexión sobre los aspectos gramaticales y discursivos del lenguaje en uso, lo que ha conducido a la elaboración de propuestas que relacionan el aprendizaje de la escritura y de la gramática mediante el fomento de la actividad metalingüística. Los resultados de investigaciones en contexto de aula realizadas por el grupo GREAL (Grupo de investigación sobre la enseñanza de las lenguas, de la Universitat Autònoma de Barcelona) muestran cómo niños y niñas de Primaria son capaces de hablar sobre la lengua si tienen un entorno que lo incentive (Milian, 2001; Gil, 2011; Casas, 2014). Otros trabajos (Calil y Myhill, 2020) concluyen, además, que los contextos colaborativos en escritura permiten al profesorado acceder al pensamiento de niños y niñas para así fundamentar mejor sus intervenciones didácticas y proporcionar un retorno ajustado a las necesidades de los alumnos.

En este artículo, basándonos en los postulados vigotskianos, concebimos la entrevista como un entorno favorable para la activación del razonamiento y para la construcción conjunta del conocimiento metalingüístico. El entrevistador, que forma parte del dispositivo diseñado para la investigación, desarrolla el rol de andamiaje para 
permitir que el alumno exprese verbalmente su reflexión. A pesar de todo -como se verá más adelante- promover estas conversaciones e interpretar las verbalizaciones de los escolares no siempre resultan tareas fáciles, especialmente en Primaria en que la capacidad de razonamiento abstracto está todavía en fases iniciales.

Los resultados de este estudio adquieren un indudable interés para la enseñanza y el aprendizaje ya que, si bien el entorno de investigación tiene unas características distintas de los contextos habituales de enseñanza, los hallazgos aportan ideas cruciales para la metodología de aula. Organizar situaciones de conversación metalingüística con los estudiantes, desde las primeras edades (Gil y Bigas, 2009; Gil, 2011), permite que se generen espacios intermentales, para pensar con otros, que ayuden a la autorregulación y la autonomía del aprendiz (Mercer, 2013).

Así pues, los objetivos de este artículo son dos:

a. Profundizar en las características de la actividad metalingüística de los alumnos de Primaria como elemento clave para el aprendizaje lingüístico, en un entorno escolar de aprendizaje de la escritura y de la gramática.

b. Mostrar las singularidades de la entrevista como espacio de diálogo entre estudiantes y adulto que permite hacer emerger la reflexión metalingüística.

\section{1.- Marco teórico}

\section{1.- El aprendizaje de la escritura y la reflexión sobre la lengua}

Nuestra investigación se sitúa en el campo científico de la Didáctica de la lengua. Tiene en cuenta principalmente las aportaciones de la psicología cognitiva que, en sus modelos de los años 80, definieron la escritura como un proceso cognitivo complejo (Flower y Hayes, 1981; Bereiter y Scardamalia, 1983) compuesto por subprocesos que tienen lugar de forma recursiva guiados por una instancia de control; considera asimismo las aportaciones de las teorías socioculturales, que consideran la escritura como una actividad socialmente situada que adquiere sentido dentro de la comunidad 
(Camps, 2017). Para aprender a escribir es preciso ayudar a descubrir este sentido a través de una instrucción explícita (Calil y Myhill, 2020).

El trabajo se inscribe en los estudios que plantean la enseñanza reflexiva de la lengua como una vía necesaria para desarrollar competencias en el ámbito de la escritura (Camps, 2003; Barbeiro y Brandão, 2006; Rodríguez-Gonzalo, 2012; Ribas, Fontich y Guasch, 2014; Myhill y Jones, 2015; Camps y Ribas, 2017; Myhill, Watson y Newman, 2020). La conciencia sobre el material lingüístico que se observa y manipula, a la vez que sobre los procesos y entornos en los que lo utilizamos, aporta estrategias y conocimientos para un uso más eficaz del lenguaje. Distintos autores destacan el papel de la metacognición en el proceso de escritura: algunos de sus componentes, como los gráfico-espaciales o los ortográficos, necesitan ser automatizados para alcanzar una buena competencia en escritura, mientras que otros requieren decisiones conscientes y capacidad de selección (Myhill et al, 2020). Incluso los aspectos automatizados pueden volverse conscientes cuando se desea un efecto especial en el lector o se reformula la finalidad del texto (Barbeiro y Brandão, 2006). Si bien quedan cuestiones por esclarecer, como la relación entre el conocimiento metalinguístico declarativo y el procedimental (Camps y Milian, 1999; Myhill y Jones, 2015), podemos decir que en la producción de textos es inevitable la toma consciente de decisiones sobre la lengua en contexto.

Estudios en didáctica de la lengua (como los recogidos en Ribas et al, 2014) plantean el desarrollo de la competencia metalinguística de los alumnos mediante un aprendizaje reflexivo de la lengua. Entendemos la reflexión como un proceso de toma de conciencia sobre el funcionamiento de la lengua (Rodríguez-Gonzalo y Durán, 2019), que no consiste en enseñar conceptos para después ser aplicados por el alumno en la escritura, sino en propiciar que sea el alumno quien construya de forma recursiva los conceptos sobre gramática y sobre el discurso mediante la manipulación de material lingüístico y la reflexión organizada sobre los usos en contextos con sentido.

Ahora bien, la práctica reflexiva de niños y niñas no aparece espontáneamente. Del mismo modo que la escritura, esta práctica se 
aprende en contextos escolares y por tanto debe ser enseñada y guiada en tareas situadas y mediante el uso de conceptos gramaticales y discursivos (Myhill y Jones, 2015; Dolz y Gagnon, 2018; Camps y Fontich, 2020). Con el fin de generar interacción para activar la conciencia metalingüística, se plantean tareas de escritura colaborativa o actividades de facilitación procesual (Barbeiro y Brandão, 2006, p. 87), en las que el profesorado aporta ayudas y los niños y niñas asumen la responsabilidad compartida en la tarea de escritura grupal hasta alcanzar su objetivo. Otro aspecto que debe ser facilitado en Primaria es la atención a los niveles más globales del texto (Calil y Myhill, 2020). La investigación sociocultural defiende que los aspectos locales relacionados con la transcripción pueden desarrollarse paralelamente a los de tipo compositivo más global (Dyson, 2009). Los estudios en esta línea apuntan que se trata de prácticas poco habituales en las aulas (Fontich y García-Folgado, 2018), especialmente en las primeras edades, pero que si se enseña a escribir de esta forma se promueve una actitud mucho más reflexiva sobre la escritura y sobre el proceso de aprendizaje, que revierte en el fomento de la actividad metalingüística y en la mejora de la competencia escrita (Calil y Myhill, 2020).

Un tema de debate en la investigación es si es necesario disponer de conocimiento gramatical previo para poder "hablar" o reflexionar sobre la lengua. Fontich y García-Folgado (2018), basándose en los supuestos vigotskianos, son taxativos: el conocimiento gramatical no debe ser una condición para reflexionar sobre la lengua sino una consecuencia. Camps (2000) ya mostró que la actividad metalingüística se puede verbalizar de distintas formas y que los conceptos gramaticales y sobre el discurso se construyen en un proceso de reformulación que contrasta y relaciona constantemente las experiencias de los alumnos como usuarios del lenguaje escrito con el marco que explica el funcionamiento del lenguaje.

Así, el dominio progresivo del metalenguaje se considera necesario como un paso hacia la conceptualización (Barth, 2004). Sin metalenguaje se hace difícil e impreciso hablar y discutir sobre la lengua y la autorregulación en el proceso de escritura no puede desarrollarse de manera satisfactoria (Camps, 2000; Myhill y Jones, 2015). En la progresión que va del reconocimiento de un fenómeno 
lingüístico hasta la elaboración explícita del concepto que lo explica, el uso de términos específicos para nombrar los fenómenos se vuelve necesario según la capacidad metalinguiística se va desarrollando.

\section{2.- La entrevista como espacio de reflexión compartida}

Entendemos la entrevista como una práctica social y discursiva (Mondada, 2001; Mann, 2011; Talmy, 2011; Guasch y Ribas, 2013) donde el discurso se construye conjuntamente entre entrevistador y entrevistado lo que permite crear un contexto de reflexión compartida post-tarea con el pequeño grupo de alumnos que han trabajado juntos (Arseneau, 2016). En el diálogo con el experto o entre iguales, los individuos ponen en marcha mecanismos autorreguladores mediante procesos de alta dificultad cognitiva como la planificación o la resolución de tareas (Vigotski, 1988). Así, el diálogo entre investigador y escolares permite observar el pensamiento en voz alta de los estudiantes y a la vez lo posibilita (Camps et al, 2001; Durán, 2010).

Lantolf (2000) -siguiendo a Vigotski- señala que para que haya aprendizaje es necesario que se dé una apropiación o interiorización de los contenidos y que ello se realice a través de las llamadas lengua interior (inner speech) y lengua privada (private speech), que tienen una función psicológica intrapersonal y cognitiva. Aparecen sobre todo cuando el estudiante se enfrenta a una tarea de alta dificultad, a un reto que le obliga a movilizar sus conocimientos previos. La lengua privada tiene una función estratégica: representa una exteriorización de las órdenes internas (lengua interior) como manera de controlar las funciones cognitivas para realizar la tarea. A través de la lengua privada la conciencia puede ser investigada, ya que permite observar las acciones que van de lo intramental a lo intermental y hace visible la lengua interior (Lantolf y Appel, 1994). Este aspecto es importante para entender la necesidad de la interacción para hacer emerger la reflexión metalingüística: para conseguir la autorregulación y para tener evidencias observables de esta lengua interior que refleja la conciencia del hablante y la construcción del conocimiento. El reto que suponen las preguntas de la investigadora provoca que los niños y niñas piensen en 
voz alta, exterioricen la lengua interior, lo que permite vislumbrar dificultades, pero también momentos de aprendizaje (Durán, 2010).

Con alumnos, la entrevista ha permitido conocer los procesos de composición escrita que desarrollan cuando componen un texto y observar su capacidad de razonamiento metalingüístico en las decisiones tomadas. Encontramos trabajos en esta línea en el grupo GREAL (Torralba, 2012; Casas, 2014, para Primaria; Camps et al, 2001; Durán, 2010 y 2020, para Secundaria, entre otros); también en el ámbito francófono, donde las entrevistas en Primaria exploran principalmente el aprendizaje de la ortografía (Jaffré, 2003; Hassan, 2004) y en menor medida el de la gramática (Arseneau, 2016). Asimismo, el equipo de la Universidad de Exeter ha realizado entrevistas con alumnos de estas edades usando la estrategia de la "estimulación del recuerdo" (stimulated recall) para comprender los procesos que siguen los alumnos en las tareas de escritura. En nuestro trabajo, buscamos que los alumnos activen su conocimiento mediante la reflexión y lo hagan en parte visible en las entrevistas, como "pequeñas experiencias comunicativas" (Torralba, 2012) cuidadosamente creadas por el equipo investigador.

\section{2.- Metodología}

La investigación que presentamos, de carácter cualitativo, trabaja con datos obtenidos en entrevistas a 17 parejas o tríos de alumnos de cuatro centros educativos públicos ${ }^{1}$, en el marco de una SD diseñada y pilotada previamente por el equipo investigador y llevada a cabo durante 11 sesiones por las maestras habituales del aula tras una fase de formación.

El tipo de entrevista realizada se conoce como "semiestructurada" o "conversación orientada" (Palou, 2008), porque se planifica en función de los objetivos de la investigación y establece una conversación organizada, iniciada y dirigida por el investigador. En

${ }^{1}$ Las SD y las entrevistas se realizaron en catalán, lengua de la escuela en Cataluña. Para el artículo, los datos se han traducido al castellano. 
nuestro caso, se retorna sobre las tareas realizadas en clase y tiene lugar inmediatamente después, usando los materiales elaborados en el aula.

El adulto desarrolla diversas estrategias para crear un clima de confianza y estimular respuestas. Los datos obtenidos por este procedimiento se consideran representaciones socialmente construidas, fruto de la colaboración en un momento determinado, y permiten comprender algunos rasgos del proceso del alumno. Según Hassan (2004), además de dar cuenta de los efectos del discurso del investigador sobre los alumnos y de los implícitos de ambos en la conversación, debemos abandonar la ilusión de poder obtener de los niños explicaciones traducibles directamente en términos de conceptualización. Sus comentarios, como veremos en nuestro estudio, pueden ser a veces poco elaborados, otras poco espontáneos, incluso convencionales, por lo cual la tarea de interpretación no es fácil para el investigador, que normalmente necesita contrastar con otros datos como las producciones escritas de cada alumno o bien con las interacciones orales mientras escriben en grupo.

En esta investigación adoptamos la entrevista con el alumnado como instrumento metodológico para observar su capacidad de reflexión metalingüística en la tarea de escritura y para acercarnos a su pensamiento sobre la lengua en una situación interactiva con el adulto. Así constatamos una vez más, siguiendo trabajos anteriores de GREAL, el valor de la conversación como espacio para reflexionar sobre la lengua (Camps et al, 2001; Milian, 2005).

La SD llevada a la práctica tenía por objetivo enseñar a escribir textos de opinión, atendiendo a la planificación del texto y a los mecanismos de autorregulación del proceso de escritura. Se experimentó con dos versiones de SD: en una, el trabajo estaba centrado solamente en estos mecanismos de autorregulación del proceso (en adelante, SD1); en la otra, se trabajaban de forma explícita, además de los contenidos mencionados, algunos elementos de conexión como organizadores discursivos y conectores causales (en adelante, SD2). En ambos casos la SD estaba guiada por el objetivo final: escribir un texto de opinión elaborado en parejas o tríos y publicado en un blog. 
Para las estrategias de planificación, se proporcionó a los alumnos un esquema textual con un acrónimo fácilmente memorizable, que aludía a los distintos componentes del texto (PER -Pienso, Explico, Resumo-para $2^{\circ}$ curso; CREC -Creo, Razón, Explicación, Conclusiónpara $4^{\circ}$ ). En la SD2, el esquema contenía además posibles conectores para introducir cada parte del texto.

Al terminar la sesión $3^{\mathrm{a}}$ o $4^{\mathrm{a}}$ ( eegún el tipo de SD) y la sesión $8^{\mathrm{a}}$, un miembro del equipo investigador mantuvo una entrevista con tres pequeños grupos (parejas o tríos) de cada aula, con preguntas que incitaban a recordar el proceso seguido, justificar las decisiones tomadas y valorar las dificultades encontradas.

Los datos analizados para el presente estudio corresponden a las entrevistas con varias parejas o tríos de alumnos de 6 aulas distintas de $2^{\circ}$ y $4^{\circ}$ curso de Primaria. Los nombres de los centros así como los de alumnos y alumnas son ficticios.

\section{Tabla 1}

Participantes en el estudio

\begin{tabular}{lccc}
\hline Escuela & Nivel & Tipo de SD & N $^{\circ}$ de parejas o tríos \\
\hline Llevant & $2^{\circ}$ Primaria & 3 \\
Llevant & $4^{\circ}$ Primaria & $\begin{array}{c}\text { SD1: proceso de escritura y } \\
\text { estrategias de autorregulación }\end{array}$ & 3 \\
Art & $2^{\circ}$ Primaria & $2^{\circ}$ Primaria & $\begin{array}{c}\text { SD2: proceso de escritura y } \\
\text { estrategias de autorregulación }+\end{array}$ \\
Ponent & $4^{\circ}$ Primaria & $\begin{array}{c}\text { reflexión explícita sobre los } \\
\text { elementos de conexión }\end{array}$ & 3 \\
Ponent & $2^{\circ}$ Primaria & 3 \\
Barcino & &
\end{tabular}

$\mathrm{n}=17$ parejas o tríos

$\mathrm{n}=40$ alumnos y alumnas

Fuente: elaboración propia

Las entrevistas se registraron en audio y posteriormente se transcribieron siguiendo los criterios del grupo Greal ${ }^{2}$. Los protocolos

${ }^{2}$ Criterios de transcripción: secuencia tonal terminal ascendente $\uparrow$, descendente $\downarrow$; pausa breve hasta 2 seg. (.), mediana 3 seg. (..), larga más de 3 seg. (...); palabra o 
transcritos se analizaron con ayuda de una pauta de codificación con categorías y subcategorías establecidas por las investigadoras a partir de la lectura recursiva y con un procedimiento de verificación interjueces. La pauta incluía también ejemplos de cada categorización, lo cual permitía con facilidad volver a los datos para verificar las interpretaciones.

Se establecieron dos tipos de categorías: las temáticas, para apreciar el contenido de los comentarios y las discursivas, relacionadas con las características propias de la comunicación dialógica. Con la combinación de ambas, para cada pareja se elaboró una síntesis final que permitió la comprensión del intercambio. Tras contrastar los análisis de las distintas parejas o tríos entre el equipo investigador, se procedió a la interpretación de las entrevistas.

\section{3.- Resultados y discusión}

Presentamos en primer lugar los resultados del análisis de contenido de las entrevistas en relación con las categorías temáticas, que muestran la reflexión metalingüística que el alumnado es capaz de realizar con la mediación del experto. A continuación, nos centramos en la entrevista como espacio discursivo que permite crear esa zona de reflexión compartida, una vez realizada la tarea.

\section{1.- Las concepciones de los alumnos manifestadas en las entrevistas 3.1.1.- La reflexión sobre los procesos de escritura}

Los datos revelan que los niños y niñas de segundo y de cuarto de Primaria del estudio son capaces de reflexionar sobre los procesos implicados en la escritura de un texto en colaboración y verbalizan sus concepciones sobre ella de una manera fluida, lo que permite inferir que se han apropiado de dichos procedimientos. Ante las preguntas de los investigadores, aluden a los beneficios y dificultades que implica la escritura en colaboración, a las estrategias para estructurar el texto a

enunciado truncado: - ; solapamientos / / ; interrupción que implica cambio de turno: $==$; texto incomprensible: XX; comentarios del transcriptor: [ ]; texto afectado por risas $\{@\}$, por volumen alto $\{\mathrm{F}\}$, por volumen bajo $\{\mathrm{P}\}$. 
partir del acrónimo utilizado y de los conectores que introducen las distintas partes, y a las diversas funciones del borrador en contraposición al texto definitivo. Lo hacen de una manera asertiva, aportando argumentos para defender sus opiniones. Solo cuando se refieren a la conclusión del texto de opinión que han escrito muestran dificultades para explicar en qué consiste ${ }^{3}$.

Veamos un ejemplo de cómo conceptualizan las diferencias entre el texto definitivo y el borrador -uno de los aspectos en que focaliza la SD- y de cómo expresan estas diferencias en las entrevistas de segundo y de cuarto curso.

${ }^{3}$ Ver Ribas, Rodríguez-Gonzalo y Durán (2020) para los conceptos de estos mismos alumnos sobre la escritura en el aula. 
A. $2^{\circ}$ Llevant, pareja A, sesión 8 (SD1)
B. $4^{\circ}$ Ponent, trío $\mathrm{C}$, sesión 8 (SD2)

31. ENTR: en sucio $\downarrow$ (..) me- (..) y para qué nos ehh sirve hacer un borrador $\uparrow$ nos ehh sirve para escribir un texto mejor hecho $\uparrow$ 32. L: sí $\downarrow$ (.) porque en el borrador $\mathbf{s i}$ haces faltas de ortografía y te das cuenta $\uparrow$ después en el limpio $\uparrow$ no las harás $\downarrow$ porque ya pensarás en ellas $\downarrow$

33. ENTR: bien $\uparrow($.$) más cosas \uparrow$ 34. G: sí $\downarrow$ para pensar $\uparrow$ las idea::s (.) para colocarlas $(-) / \mathrm{y}$ después $=/$

35. ENTR: /eso es muy/ importante $\downarrow$ 36. G: =aquí $\downarrow$ ehh: haces como un resumen (.) poniéndolo mejo::r 37. L: y aquí si te equivocas y (.) y piensas que:: te quedará mejor de otra manera lo puedes borrar tanto como quieras aunque quede sucio porque aqui [en el texto final] ya harás lo definitivo $\downarrow$ un borrador $\uparrow$
25. M: /para pensar las/ideas $\downarrow$
26. I: /para pensar todo/
para plasti(.)ficar-
27. ENTR: planificar muy bien $\uparrow$
28. I: planificar todo y ha- (.) ponerlo en otro papel $\downarrow$
29. ENTR: y tú decías las ideas $\uparrow$ (.) para hacer qué con las ideas $\uparrow$
30. $\quad \mathrm{M}: \mathrm{eh} \uparrow($.) el papel $\downarrow$
31. ENTR: tú decías \{el borrador sirve para las ideas $\downarrow$ \}
para hacer qué con las ideas $\uparrow$
32. M: pues pa:ra primero pensamos $\uparrow$ y lo ap- (.) y
después (.) si hemos pensado todas las ideas $\uparrow$ nos las
apuntamos $\uparrow$ (.) después las ponemos allá $\downarrow$ (...) y::
33. ENTR: allá quieres decir en el borrador $\uparrow$
34. M: sí $\downarrow$

En estos fragmentos, los escolares entrevistados muestran que son capaces de observar distintas funciones del borrador. Por un lado, señalan que les ayuda a "pensar las ideas", a planificar, es decir, el borrador les permite concentrarse en aquello que quieren decir, sin preocuparse por la forma en que lo dicen, lo que supone una importante descarga cognitiva. Por otro lado, les ayuda a revisar, a regular la escritura: "si haces faltas de ortografía y te das cuenta [...] después en el limpio no las harás porque ya pensarás en ellas" (ejemplo 1B, turno 32). La misma función del borrador como regulador de la escritura aparece de nuevo más adelante: "si te equivocas y piensas que te quedará mejor de otra manera" (1B, t.37). 
En las entrevistas a $2^{\circ}$ de Primaria, aparecen con frecuencia alusiones a aspectos materiales, como la extensión o la presentación (1A, t.12-14), lo que coincide con los hallazgos de Calil y Myhill (2020) en que la prioridad de los jóvenes escritores está en los aspectos gráficos y espaciales (p. 13).

Tanto para los escolares de $2^{\circ}$ como de $4^{\circ}$, el borrador no presenta grandes diferencias de contenido, pero cumple un importante papel regulador en relación con las ideas - "si hemos pensado todas las ideas, nos las apuntamos" (1A, t.32; 1B, t.34)-, en la forma de decirlas (1B, t.37) o en escribir las palabras correctamente (1B, t.32). El borrador les permite volver conscientemente sobre aquello que desean decir, y pensar en la forma de expresarlo.

En definitiva, los niños muestran en sus respuestas que son capaces de pensar sobre las funciones del borrador explicándolas con sus propias palabras. Pueden pensar sobre los procesos de escritura (sobre la planificación, la textualización y la revisión) a partir del ejemplo de su propio texto, un objeto de reflexión concreto del que pueden hablar y al que se pueden referir con los términos adecuados. Muestran, por tanto, que están desarrollando una comprensión metalingüística sobre la escritura y, en ese proceso, el diálogo con el adulto crea el contexto adecuado para tomar conciencia de ello e ir articulando y justificando su propio pensamiento.

\subsection{2.- Hablar sobre la lengua: los conectores}

Las SD diseñadas, tanto en $2^{\circ}$ como en $4^{\circ}$ de Primaria, solo se diferenciaban por el papel que concedían a la reflexión explícita sobre los conectores ${ }^{4}$. En la SD2 se trabajaba sobre los conectores de causa y

${ }^{4}$ En este trabajo se utiliza el término conector entendido, según el Glosario de términos gramaticales, como "Unidad léxica, formada por una o varias palabras, que establece vínculos lógicos, discursivos o argumentativos entre los segmentos de un texto.” (RAE-ASALE, 2019, pp. 74-75). La clasificación más frecuente organiza los conectores en tres grupos: argumentativos, reformuladores y estructuradores. En nuestra $\mathrm{SD}$, solo se trabajaban algunos conectores argumentativos (porque, ya que...) y los estructuradores de organización discursiva (en primer lugar...). 
consecuencia (porque, ya que...) y de organización del texto (en primer lugar, en segundo lugar, en conclusión...), lo que implicaba el uso del término conector para referirse a ellos. En la SD1 no se realizaban actividades explícitas sobre ellos ni se utilizaba el término como tal, aunque en los textos de los alumnos se podían observar usos espontáneos de palabras de este tipo.

Esta diferencia tuvo implicaciones en las entrevistas. Los entrevistadores solo preguntaron explícitamente por los conectores en la SD2, mientras que, en la SD1, las alusiones fueron ocasionales, al hilo de la reflexión sobre distintos aspectos de la escritura. En ambos casos, la interpretación de las respuestas de los alumnos requiere tener en cuenta las preguntas de los entrevistadores.

En las entrevistas a los grupos de SD2, encontramos dos tipos de situaciones en que los alumnos hablan de los conectores usando explícitamente el término: a) a instancias del entrevistador, que pregunta para qué sirven, qué son, cómo se llaman estas palabras y b) de manera espontánea, mientras los alumnos explican diversos aspectos del texto que han escrito o cuando aluden a aquello que más les ha gustado aprender. En las entrevistas a los grupos SD1, en cambio, aparecen alusiones a la función o al significado de los conectores empleados en los textos de los alumnos siempre de manera indirecta, señalando con el lápiz las palabras, ya que el término conector no había sido introducido como tal en la SD y, por tanto, los entrevistadores no lo podían usar en sus preguntas.

a. Reflexiones de los alumnos a instancias de las preguntas explícitas del entrevistador sobre los conectores (SD2)

La pregunta sobre la función de los conectores (para qué os han servido) vincula directamente estas palabras con el uso que los alumnos han hecho de ellas al escribir sus textos de opinión. Observamos respuestas como las recogidas en los siguientes ejemplos: 


\section{Ejemplo 2}

A. $2^{\circ}$ Barcino, pareja C, sesión 4 (SD2)

158.ENTR: \{(@) muy bien $\}$ (.) muy bien y- (.)y qué habéis aprendido hasta ahora de los conectores $\uparrow$ qué diríais $\uparrow$ para qué sirven $\uparrow$

159. Y: para- para para-

160. L: para hacer un texto de o-de o- de- (.) un texto de buen-

161. Y: de opinión bien he-

162. L: $\{(\mathrm{F})$ un buen texto de- de $\{(@)$ buena opinión $\}$ [...]

168. Y:/pero porque / em: para e- em: con ellos si estoy hablando con una persona que es más grande que(.) que- que yo le- (.) le tendría que hablar no como una niña pequeña sino como e- como ellos dirían en(.) en buenos textos de opinión $\downarrow$
B. $4^{\circ}$ Ponent, trío B, sesión 8 (SD2)

49: ENTR: y para qué creéis que os han servido los conectores para hacer el texto

50.A: para que concuerden mejor las frases

51.ENTR: para que concuerden mejor las frases sí sí (.) para más cosas

52.TA: porque (.) sin conectores no se entendería bien la::

53.ENTR: sin conectores no se acaba de entender bien (.) e: las cosas que queremos decir no (...) se entienden mejor si ponemos conectores (...) sí

54. TA: sí

En 2A, las primeras respuestas de alumnos de $2^{\circ}$ de Primaria parecen reproducir las explicaciones recibidas ("para hacer un buen texto de opinión", t.160-161). Sin embargo, más adelante, la alumna Y aporta una reflexión propia -"si estoy hablando con una persona que es más grande que yo..." (t.168) - con la que muestra que percibe los conectores como un elemento discursivo que le permite explorar otras formas de decir, de hablar como alguien mayor que ella, de aprender a decir mejor las cosas. En sus palabras observamos la interiorización de las explicaciones de la maestra ("hacer buenos textos de opinión") y cómo la refiere a su conciencia como hablante.

También en el ejemplo 2B, de $4^{\circ}$ de Primaria, los alumnos muestran reflexiones que apuntan a una interiorización de la función de los conectores. Señalan explícitamente la importancia de los conectores como elementos de la cohesión del texto (t.50-52), "para que concuerden mejor las frases") y son capaces de explicarse con palabras propias.

La reflexión metalingüística propia emerge cuando los escolares se alejan de las respuestas esperadas (que reproducen las explicaciones de la maestra). Esta reflexión viene propiciada por el término de metalenguaje, que la activa y la facilita. Cuando la pregunta no remite a la función sino a la explicación del término conector (qué son) o a su 


\section{identificación (cómo se llaman estas palabras) las dificultades de los alumnos son mayores (3 A y B).}

\section{Ejemplo 3}

A. $2^{\circ}$ Ponent, pareja A, sesión 4 (SD2)

B. $4^{\circ}$ Ponent, trío A, sesión 4 (SD2)

152. ENTR: $[\ldots]$ nos podríais explicar bien (..) qué son los conectores $\uparrow$

153. A: pa- palabras::s $\{(\mathrm{PP})$ para:: $\}$

154. R: porque lo::s-

155. ENTR: déjala un momentito $\downarrow$ (.) a ver $\downarrow$ (.) acaba $\downarrow$ (.) palabras que $\uparrow$ (..) qué- qué tipo de palabras $\uparrow$ después nos lo explicas tú $\downarrow$ (...) muy bien muy bien $\uparrow$ (..) qué palabras son los conectores $\uparrow$

156. A: e::

157.ENTR-2: para qué sirven estas palabras $\uparrow$

158. A: /para decir lo que pienso $\uparrow /$

159. R: /yo lo sé $\downarrow /$

160. ENTR-2: $\mathrm{mmm} \uparrow$

161. R: yo lo sé también $\downarrow$

162. ENTR-2: y qué $\uparrow$

163. R: y que los (.) lec(.)tores nos entiendan $\downarrow$

[...]

239.ENTR-2: estas palabras nos ayudan a hacer alguna cosa o no $\uparrow$

240. A: sí $\downarrow$

241. ENTR-2: qué $\uparrow$

242. R: a escribir buenos textos de opinión $\downarrow$

243.ENTR-2: aaaah (.) buenos textos de opinión $\downarrow$

244. ENTR-1: y más cosas $\uparrow$ nos sirven para más cosas $\uparrow$

245. A: sí $\downarrow$

246. ENTR-2: para qué $\uparrow$ (.) a ver $\downarrow$ (.) explica $\downarrow$

247. A: $\{(\mathrm{P})$ dar tu opinión $\}$
102. ENTR: [...] estas palabras que hemos usado en aquí $\uparrow$ (...) estas del de la primera razón:: (.) el porque:: ya que:: (.) todas estas palabras que hemos utilizado $\uparrow \uparrow$ quién lo sabe cómo se llaman estas palabras que hemos utilizado $\uparrow$

103. D: em:: conectores $\downarrow$

104. ENTR: tú sabes cómo se llaman estas palabras que hemos utilizado para comenzar las diferentes partes del CREC $\uparrow$ cómo se llaman estas palabras $\uparrow \quad(\ldots)$ y tú lo sabes $\uparrow$

[...]

107. M: conectores $\downarrow$

108. ENTR: co- y V tú K explícanos $\downarrow$ (.) cómo explicarías qué es un conector $\uparrow($.) cómo nos los explicarías si nosotros no lo supiésemos

[...]

125. K: que lo diga $D \downarrow$

[...]

127. K: yo no tengo ni idea $\downarrow$

[...]

131. D: em::: la:: los conectores sirven pa::ra el que esté leyendo los textos entienda mejor lo que estamos intentando expresar y que:: em:: esté con nosotros cuando estamos haciendo texto de opinión $\downarrow$

$[\ldots]$

136. ENTR: y qué relación tienen los conectores con la estrategia del CREC $\uparrow$

137. D: que::: em::: el::: las razones necesitan tener conectores $\uparrow$ para que se entiendan bien $\downarrow$ también las explicaciones la conclusió y decir el CREC también necesitan conectores $\downarrow$

138. ENTR: muy bien y $\mathrm{K} \downarrow$ (.) cada parte (.) la cl (.) las razones (.) las explicaciones (.) la conclusión $\uparrow$ utilizan los mismos conectores $\uparrow$

139. K: no $\downarrow$ 
Tanto en $3 \mathrm{~A}$ como en $3 \mathrm{~B}\left(2^{\circ}\right.$ y $4^{\circ}$ de Primaria respectivamente) se observa que los alumnos reconocen el término conector cuando lo utiliza la entrevistadora e identifican sin dificultad los conectores en el texto que tienen delante. Pero cuando intentan explicar qué es un conector, las diferencias son notables tanto entre $2^{\circ}$ y $4^{\circ}$ como entre los propios alumnos de $4^{\circ}$. En $3 \mathrm{~A}$, la alumna $\mathrm{A}$, de $2^{\circ}$, los identifica como palabras que le permiten decir lo que piensa, para dar la opinión (t.247), por lo que parece que alude a conectores como porque o ya que. En cambio, la intervención de su compañero R (t.242) apunta a los conectores de orden, que facilitan al lector la comprensión del texto.

En $3 \mathrm{~B}$ ( $4^{\circ}$ de Primaria), K sabe que es incapaz de explicar qué entiende por conector (t.127) y es consciente de que su compañero sí sabrá hacerlo, por lo que le pasa la palabra (t.125). En cambio, cuando la entrevistadora le pregunta si todas las partes del texto utilizan los mismos conectores, tiene claro que no (t.139). Es decir, K no puede verbalizar la actividad metalingüística que realiza, de carácter manipulativo básicamente (sabe utilizar los conectores, pero no puede hablar de ellos fuera del texto). En cambio, D (t.131,137), reformulando las explicaciones de la maestra, es capaz de explicar la función de los conectores tanto para facilitar la comprensión del lector (t.131) como por requerimientos del texto ya que sabe que la estrategia CREC exige conectores para guiar las diferentes partes del texto (t.137).

En estos casos observamos diferencias en la conceptualización de conector. El uso del término permite identificarlo con facilidad, tanto a los alumnos de $2^{\circ}$ como de $4^{\circ}$, y también reconocer diferencias, como ocurre en $3 \mathrm{~B}$ (t.139) cuando $\mathrm{K}$ responde con seguridad a la pregunta de si todas las partes del texto usan los mismos conectores. La explicación, en cambio, implica un mayor grado de abstracción, ya que exige alejarse del texto y generalizar. De ahí que las preguntas directas sobre los conectores planteen dificultades a los alumnos, que solo son capaces de solventar cuando el entrevistador reconduce la pregunta hacia la función de estas palabras, como en 3A (t. 239), o cuando son los propios alumnos los que utilizan esta estrategia, como hace $\mathrm{D}$ en el ejemplo de $4^{\circ}$ de Primaria: "los conectores sirven para..." (3B, t.131). El uso del término se revela como un factor de conceptualización, en la 
medida en que permite afianzar la percepción, fase primera de la conceptualización (Barth, 2004).

b. Uso del término conector en reflexiones espontáneas de los alumnos (SD2 y SD1)

Durante las entrevistas SD2, encontramos también situaciones en que los alumnos utilizan el término conector sin que haya sido introducido por el entrevistador, como en 4 ( $2^{\circ}$ de Primaria).

\section{Ejemplo 4}

$2^{\circ}$ Ponent, pareja B, sesión 8 (SD2)

5. ENTR: vale $\downarrow$ y (.) cuando habéis hecho el texto $\uparrow$ qué cosas diferentes habéis hecho entre el borrador y el texto $\uparrow$

6. E: que que qu- que en el en el en te- texto $\uparrow$

7. M: le hemos puesto los conectores $\downarrow$

8. E: lo hemos hecho asî $\uparrow$ nd nd nd nd

9. ENTR: espera déjale que lo explique un poco ella ahora $\downarrow$

10. E: de poner PER en vertical $\downarrow$

11. ENTR: y en el texto también habéis hecho eso en vertical $\uparrow$

12. M: no $\downarrow$

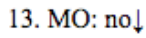

14. ENTR: no $\downarrow$ (.) cómo lo habéis hecho en el texto $\uparrow$

15. M: por porque aqui $\uparrow$ aquí no poníamos los conectores lo poníamos aquí para acordarnos $\uparrow$ de de ponerlo en en el texto $\uparrow$ y al i al y aquí poniamos si mejor o no $\uparrow$ (.) aquí las tres explicaciones $\uparrow$ sin los conectores y aquí también sin conectores $\downarrow$

En 4, el término aparece espontáneamente mientras los alumnos hablan de los textos de opinión que han escrito. Usan el término por propia iniciativa y con naturalidad cuando explican las diferencias entre la escritura del borrador (en el que no han puesto los conectores, t.15) y de su texto definitivo ("le hemos puesto los conectores", t.7). Con ello muestran que se han apropiado del término, lo que les permite hablar de unos elementos linguísticos concretos, que ya perciben como ejemplos de una categoría más amplia (conector), todavía poco perfilada. Son conscientes, además, de que su incorporación es relevante para la organización de las ideas en su texto definitivo. 
En las entrevistas a los grupos de SD1 no encontramos el término conector, ni en las preguntas de los entrevistadores ni en las intervenciones de los alumnos, ya que no había sido objeto de enseñanza explícita. Pero sí aparecen alusiones a palabras de esta categoría cuando los alumnos hablan de la escritura de sus textos, situación similar a la ya comentada. La manera de referirse a estos términos muestra las limitaciones de una reflexión metalingüística sin metalenguaje.

\section{Ejemplo 5}

$2^{\circ}$ Llevant, pareja B, sesión 3 (SD1)

\footnotetext{
44. L: y finalmente /porque es un buen lugar para hacer amigos $\downarrow /$

45. A: /porque es un buen lugar para hacer amigos $\downarrow /$

46. ENTR: y cómo lo habéis sabido que:-

47. A: porque aquí pone finalmente quiere decir que es la - la tercera:: opinión $\downarrow$ (.) /de ella $\downarrow$ /

48. ENTR: /si $\uparrow /$ finalmente quiere decir lo mismo que la tercera $\uparrow$

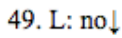

50. ENTR: qué quiere decir finalmente $\uparrow$

51. A: pues finalmente que acabas de de=

52. L: de explicar la pregunta $\downarrow$

53. A: = de explicar la pregunta $\downarrow$ y que: (.) querría decir la tercer $a \downarrow$

54. ENTR: y por qué /la tercera $\uparrow /$

55. A: /explicación $\downarrow /$

56. ENTR: y no es la cuarta $\uparrow$

57.A: porque (..) porque no hay la cuarta y:: solo hay la tercera no hay más /explicaciones/

58.L: /y si hubie/ se la cuarta tendría que pasar primero la tercera $\downarrow$
}

En 5, los alumnos comentan cómo han introducido las explicaciones para defender su postura ("y finalmente, porque es un buen lugar para hacer amigos", 5, t.44) y cómo las detectan ("porque aquí pone finalmente quiere decir que es la tercera opinión”, 5, t.47. La entrevistadora aprovecha para preguntar por el significado de finalmente y su equivalencia con la tercera opinión $(5, \mathrm{t} .48,54,56)$, lo que desencadena una interesante reflexión metalingüística de los alumnos que intuitivamente perciben el carácter deíctico del término, cuyo significado varía según los elementos que le preceden ("porque no hay cuarta y solo hay tercera, no hay más explicaciones" y L añade "y si hubiera la cuarta tendría que pasar primero la tercera" 5, t.57-58). La 
falta de metalenguaje no impide que los alumnos reflexionen sobre la lengua, pero les obliga - a ellos y a la entrevistadora- a señalar las palabras directamente en el texto, sin posibilidad de utilizar un término que las identifique $y$ que permita generalizar $y$, por tanto, conceptualizar.

La situación del ejemplo 5 muestra cómo los alumnos intentan encontrar la manera de verbalizar sus incipientes observaciones sobre las formas lingüísticas que utilizan en la escritura. La ausencia de metalenguaje se revela como una dificultad añadida para hablar de las palabras, para denominarlas en un primer grado de generalización. En el aprendizaje de la abstracción, la denominación, que no la definición, es uno de los requisitos del concepto (Barth, 2004).

Para cerrar esta presentación de resultados, remarcamos que en las entrevistas de alumnos que han trabajado con la SD2 (con reflexión explícita sobre los conectores), el uso de este término como metalenguaje les permite avanzar en las explicaciones sobre su uso, "mirar" estas palabras y pensar y hablar sobre ellas. Para los alumnos que han trabajado con la SD1, en cambio, son palabras sin identidad propia (no categorizadas), que aparecen espontáneamente en sus textos y sobre las cuales realizan reflexiones aisladas, vinculadas a la literalidad de cada caso, pero sin posibilidad de categorizar, siquiera mínimamente.

La introducción de un término gramatical con carácter manipulativo, conector en nuestro caso, permite a los escolares iniciar la conceptualización, bien diferenciando su función de la que realizan otras palabras, bien aproximándose a explicaciones de su uso, como hemos visto en los ejemplos. Se trata de usar didácticamente la denominación ("esta palabra es un verbo"), que no es lo mismo que la definición ("un verbo es una palabra que..."), como propugnaba Lenz, ya en 1912, para que los escolares aprendan a diferenciar y categorizar las palabras y puedan pensar sobre ellas. No se pretende, por tanto, que aprendan y repitan definiciones que no entienden. Como señala Barth (2004), el término o la denominación es el primer paso hacia la conceptualización, proceso que tiene un largo desarrollo (usar-designar- 
observar-primera definición-problematizar). Una primera aproximación a los términos supone usarlos, aprender a designarlos y a observarlos, lo que llevaría a una primera definición o conceptualización, que es la pretendida, a nuestro juicio, en Primaria. Esa aproximación inicial es la que permitiría en etapas posteriores que los estudiantes problematizaran ese primer concepto, es decir, que se profundizara en él, más allá de lo que ya se sabe, para avanzar hacia una definición que integrara el concepto en un sistema, lo que permitiría usarlo de forma consciente en contextos complejos.

\section{2.- La entrevista como espacio interactivo de reflexión}

La situación de entrevista busca crear un espacio de interacción metalingüística que permita a los niños hablar sobre las actividades realizadas con mayor o menor grado de reflexión. En este espacio, la atribución de funciones es muy clara: el investigador realiza preguntas para propiciar la reflexión y los entrevistados, niños y niñas de $2^{\circ}$ y $4^{\circ}$ de Primaria, se comportan como alumnos que quieren colaborar con el entrevistador, en parte, como si esperaran ser evaluados por él (de forma similar a los intercambios característicos en el aula: intervención del docente, respuesta del alumno, evaluación del docente). Esta clara distribución de funciones esconde, sin embargo, una gran complejidad por ambas partes.

El análisis de las entrevistas realizadas muestra las dificultades del investigador para encontrar preguntas que hagan emerger la reflexión de los niños, mucho más evidentes cuando no comparten terminología. Por una parte, encontramos preguntas que provocan respuestas "automáticas", aprendidas, que parecen reproducir las palabras de la docente (6A, t.24). Por otra, hay preguntas que conducen con facilidad a la simple aserción (o negación), como sucede en 6A (t.27-33) y no permiten acceder realmente a la reflexión de los alumnos. Por ello, el entrevistador se ve obligado a repreguntar, intentando que los niños aporten razones que muestren su propia manera de entender aquello de lo que se está hablando. En 6A (t.34), el entrevistador lo intenta "¿por qué estaba mejor?”, lo que provoca un cierto conflicto cognitivo en los alumnos (t.35), que no aciertan a responder. Tras otro 
intento, también fallido, decide proporcionar alguna pista (t.38) "¿os pareció que se podía leer mejor o que se entendía mejor si tenía conectores?", que sirve de apoyo a H para elegir una de las opciones proporcionadas (t.39). Las dificultades de verbalización muestran los problemas de interiorización de la noción por parte de los alumnos, que no encuentran la manera de hablar de ella con palabras propias.

A la necesidad de que el entrevistador adopte un papel estratégico para encontrar la forma de hacer emerger la reflexión metalingüística de los niños, se suma el problema de la terminología compartida. En 6A, entrevistador y alumnos comparten el término conectores, que les sirve para aludir a algunas palabras del texto. En cambio, en 6B (que corresponde a una SD1), el entrevistador ha de leer directamente las palabras (t.142, primero, en segundo lugar) y utiliza la palabra "cosas" para preguntar por ellas (t.142. "¿qué son estas cosas?"). La vaguedad del término bloquea a los niños (6B, t.143-146) hasta que la repregunta $(6 \mathrm{~B}, \mathrm{t} .147$, “¿para qué sirve?) permite a $\mathrm{N}$ evocar una situación anterior en que habían usado expresiones similares al escribir recetas $(6 \mathrm{~B}, \mathrm{t} .148,153,157)$, sin que por ello sea capaz de explicar el uso de estas palabras (6B, t.160-161 "no me acuerdo"). La insistencia y la ayuda de la entrevistadora finalmente consiguen que $\mathrm{N}$ encuentre la manera de verbalizar una primera reflexión ("porque si no, estaría todo mezclado", 6B, t.169 y 171), lo que aprovecha la entrevistadora para reformular como "está más ordenado" (6B. t.172).

Ejemplo 6

A. $2^{\circ}$ Barcino, pareja B, B. $2^{\circ}$ Art, trío B, sesión 8 (SD1) sesión 4 (SD2)

23. ENTR: $[\ldots]$ escuchad y eso a ver esto de los conectores que estáis haciendo estos días (.) eh:: para qué creéis que sirven los conectores $\uparrow$

24. H: para (.) para ayudarnos a hacer buenos textos de opinión $\downarrow$

25. ENTR: y por qué (.) por qué ayudan $\uparrow$ (..) qué pasa cuando escribimos sin conectores $\uparrow$

26. H: pasa que que que lo hacemos mal (.) y:: (.) y y acaba de de y lo (.) y
142. ENTR: y tú has escrito las dos [razones] $\downarrow$ y te has cansado un poco no $\uparrow$ de escribir las dos $\uparrow$ (.) y: ya ya ya acabamos eh $\uparrow$ (.) eso que decís aquí dice $\downarrow$ (lee) primero $\uparrow$ porque los niños $\downarrow$ (.) y entonces decís el porqué (.) (lee) en segundo lugar $\uparrow$ (.) qué son estas cosas $\uparrow$ (.) prime:ro (.) en segundo lu:gar

143. N: em:: so:n:

144. ENTR: eso lo habéis hablado con D [la maestra] $\uparrow$

145. N: sí sí

146. P: no yo sé $\downarrow$

147. ENTR: no habéis hablado $\downarrow$ (.) para qué sirve $\uparrow$ 
sa y (.) le (.) y lo habíamos y lo e:: la (..) y lo habíamos hecho sin pensar $\downarrow$

27. ENTR: y:: y eso de pensar qué (.) qué os parece $\uparrow($.) está bien pensar los textos $\uparrow$

28. H: sí

29. AI: sí

30. ENTR: sí $\uparrow$ (..) pensar un poco lo que queréis decir:: (.) y con los XXX (.) el otro día hacíamos un ejercicio con un texto que no tenía conectores (.)

\section{D: sí}

32. ENTR: no $\uparrow$ (.) y después otro que sí que tenía $\downarrow$ (.) y:: estaba mejor o no $\uparrow$ con (.) con conectores (.) el texto $\downarrow$

33. H: eh:: bien (.) / XXX/y no

34. ENTR:

$/$ sí $\uparrow /(\ldots)$ por por qué por qué estaba mejor $\uparrow$ (.) si os parece que sí $\uparrow$

35. A, H, D: ss::

36. ENTR: je je (ríe porque no contestan) (..) sabéis por qué o no $\uparrow / 0$ tenéis una $=$ /

37. $\mathrm{H}: \quad /$ no /

38. ENTR: $\quad=$ idea $($.$) no \uparrow($.) os pareció que se podía leer mejor:: o que se entendía mejor $\uparrow$ (.) si tenía conectores $\uparrow$

39. H: que se entendía mejor $\downarrow$

40. ENTR: sí $\uparrow$

41. AI: sí

42. ENTR: os pareció que sí
148.N: es por la: como lo hemos hablado en la en: la receta

149.ENTR: en la receta lo habéis hablado $\uparrow$

150. N: sí

151. AN: ah sí $\downarrow$

152. ENTR: y (.) sí $\uparrow$ (.) y para qué servía $\uparrow$ para qué sirve $\uparrow$

153. N: para decir primero $\downarrow$ (.) en primer lugar $\uparrow$ em:: (..) como

154. ENTR: en primer lugar $\uparrow$ (.) pondremos el agua al fuego $\downarrow$ por ejemplo $\downarrow$ no $\uparrow$

155. N: sí $\downarrow$

156. ENTR: y después-

157. N: y en segundo lugar $\uparrow$ ponemos las patatas

158. ENTR: $\mathrm{mmm} \uparrow($ (.) y eso y eso cuando cuando cuando (.) cuando lo utilizáis en un texto escrito $\uparrow$ (.) qué qué hace $\uparrow$

159. N: ehh::

160. ENTR: para qué sirve $\uparrow$

161. N: pa:ra (.) ehh:: (.) no me- no me acuerdo $\downarrow$

162. ENTR: no te acuerdas $\downarrow$ pero bueno para qué diríais que sirve $\uparrow$ para que se pueda entender: (.) peor $\uparrow$

163. N: no $\downarrow$

164. AN: no

165. ENTR: o para que se pueda entender mejor $\uparrow$

166. N: mejor $\downarrow$

167. AN: sí $\downarrow$

168. ENTR: sí $\uparrow$ (..) aquí si decís en primer lugar $\uparrow$ (.) todos los pa-

169. $\mathrm{N}$ : porque si no estaría todo mezclado $=$

170. ENTR: claro

171. $\mathrm{N}:=\mathbf{y}$ así es mejor porque no estaría mezclado $\downarrow$

172.ENTR: mmm (..) porque así el texto no está tan mezclado $\downarrow$ (.) está más ordenado $\downarrow$ (.) no $\uparrow$

173. N: sí $\downarrow$

En las entrevistas con escolares también son frecuentes momentos de bloqueo, cuando el entrevistado no sabe qué contestar, como observamos en 7. Se trata en este caso de preguntas que no admiten respuestas asertivas (t.108 y 110) porque intentan hacer emerger la reflexión metalingüística de forma verbalizada, lo que no resulta fácil ni posible para buena parte de escolares. 


\section{Ejemplo 7}

$4^{\circ}$ Ponent, trío A, sesión 4 (SD2)

108. ENTR: co- a ver tú Kevin explícanos $\downarrow$ (.) cómo explicarías qué es un conector $\uparrow$ (.) cómo nos lo explicarías si nosotros no lo supiésemos $\uparrow$

109. K: pue:::s

110. ENTR: qué nos dirías que es $\uparrow$ [dirigiéndose a otro miembro del grupo que quiere intervenir] a ver si él lo sabe y después lo explicas tú $\downarrow$

111. K: $\mathrm{m}:$ :

112. ENTR: cómo lo dirías $\uparrow$ (..) si le has de explicar a un niño de primero $\uparrow$ cómo lo explicarías $\uparrow$ (...5 segundos) cómo lo explicarías $\uparrow$ (...4 segundos) ni idea $\uparrow$

Como hemos visto, las entrevistas reflejan el esfuerzo de entrevistador y entrevistados por buscar un terreno compartido en el que hablar sobre la lengua. Las preguntas desempeñan un papel fundamental, ya que, para el entrevistador, la dificultad radica en encontrar formas de hacer emerger la reflexión metalingüística de los alumnos. Para que esta reflexión aparezca, lo que no siempre se consigue, es necesario que el entrevistador sea paciente, que no invada el tiempo de sus interlocutores y que propicie un diálogo en el que quepan las dudas y los titubeos. Para los niños, por su parte, la dificultad consiste en aprender a hablar sobre la lengua, que es, al mismo tiempo, una manera de aprender a reflexionar sobre ella.

Las características discursivas de estas entrevistas muestran la dificultad de articular espacios en que los escolares verbalicen la actividad metalingüística que realizan al escribir. La tarea del entrevistador resulta compleja. Ha de buscar estrategias que faciliten a los estudiantes hablar sobre aquello que han hecho, no para dar cuenta de ello sino para reflexionar, fuera del contexto convencional del docente que evalúa las respuestas de los alumnos. Salir de esta dinámica desconcierta a los escolares que, sin embargo, en muchas ocasiones consiguen esbozar reflexiones de mucho interés, en línea con trabajos anteriores sobre reflexión metalingüística en Primaria (Gil y Bigas, 2009; Gil, 2011; Casas, 2014). 


\section{Conclusiones e implicaciones didácticas}

En relación con los objetivos planteados, sintetizamos ahora nuestras conclusiones en torno a dos grandes apartados: a) la reflexión metalingüística de los alumnos de Primaria sobre la escritura y la lengua, en particular sobre el uso de conectores en textos de opinión y b) la entrevista como espacio de reflexión sobre la lengua.

a. La reflexión metalingüística de los alumnos de Primaria

Los alumnos de Primaria, tanto de segundo como de cuarto curso, se muestran capaces de verbalizar sus reflexiones sobre la lengua pero con grandes diferencias según el objeto en que se centre esta reflexión. Cuando se trata de hablar sobre la escritura, actividad que los alumnos han realizado en colaboración y pautada en distintas acciones (pensar y organizar las ideas, escribir un borrador, revisar textos ajenos, redactar el texto final), la conversación resulta fluida, ya que la experiencia vivida proporciona asideros para la reflexión y los alumnos son capaces de razonar y de explicar las dificultades y los beneficios que han observado durante su realización. Son conscientes, por ejemplo, del valor regulador de elementos como el borrador, que les sirve tanto para planificar el contenido del texto como para revisar su escritura.

En cambio, la reflexión sobre la lengua, centrada en este caso en los conectores, está vinculada a un proceso de conceptualización, por lo que la conversación se centra en la función de estas palabras o en la explicación de sus características o de sus usos. El reconocimiento del término y la función vinculada a los usos son los primeros pasos para la construcción del concepto. Solo los escolares que han trabajado de forma explícita con estos elementos (SD2) y han aprendido a denominarlos al tiempo que los han utilizado en sus textos son capaces de hablar sobre ellos, si bien con muchas dificultades, más acusadas en $2^{\circ}$ que en $4^{\circ}$ de Primaria. Poder hacer uso de metalenguaje facilita la observación de la lengua y permite hablar sobre ella, lo que implica iniciar el proceso de conceptualización del conocimiento gramatical. 
b. La entrevista, como espacio de reflexión compartida

Las entrevistas se muestran como un espacio de reflexión compartida que activa el razonamiento metalingüístico de los escolares y que permite asimismo a los investigadores acceder a este razonamiento. La conversación orientada ayuda al alumno a pensar sobre lo que ha hecho y a justificar sus ideas. Para ello, el adulto tiene que preguntar de forma que desencadene razonamiento en el alumno, lo que no siempre resulta fácil, como hemos mostrado. A partir de estas preguntas se ponen de manifiesto las diferencias de exigencia cognitiva en la reflexión. Más allá de las respuestas que reproducen con seguridad el discurso del docente, son los titubeos y los silencios, como manifestación de las dudas, los que anuncian la reflexión propiamente dicha.

Las entrevistas muestran que los niños y niñas son capaces de reflexionar sobre la escritura y sobre la lengua desde edades tempranas (Gil, 2011; Calil y Myhill, 2020), con diferencias esperables según el nivel. Son más explícitas y completas las verbalizaciones en $4^{\circ}$ que en $2^{\circ}$ de Primaria, pero también hay diferencias notables en un mismo curso, especialmente en relación con la capacidad de abstracción de cada alumno.

Pese a que las entrevistas se realizaron con fines de investigación, las implicaciones didácticas son evidentes. La reflexión metalingüística ha de ser objeto de atención didáctica en el aula. Para ello es necesario crear espacios de diálogo sobre la lengua, en un clima de conversación y confianza, opuesto a los roles tradicionales del profesor que pregunta para evaluar y del alumno que debe acertar la respuesta correcta. Se trata de que los alumnos, desde las primeras edades, adquieran hábitos de reflexión compartida, en conversaciones guiadas por el docente en las que aprendan a justificar sus ideas, a reflexionar y a verbalizar sus reflexiones. Para ello, también sería necesario contemplar este aspecto en la formación de docentes, es decir, enseñar a los docentes a gestionar este tipo de conversaciones. 


\section{Referencias bibliográficas}

Allal, L. (1993). Évaluation formative des processus d'apprentissage: le rôle des régulations métacognitives. En R. Hivon (dir.), L'évaluation des apprentissages - Réflexions, nouvelles tendances et formation (pp. 57-74). Sherbrooke, Canadá: Ed. du CRP.

Arseneau, R. (2016). Coopérer pour apprendre la syntaxe écrite: recherche-action sur la phrase subordonnée relative en troisième secondaire. Tesis doctoral. Université de Montréal. Recuperado

de https://papyrus.bib.umontreal.ca/xmlui/handle/1866/18595

Barbeiro, L., y Brandão Carvalho, J.A. (2006). Enseñar y aprender a escribir en la escuela: algunas líneas de investigación. En A. Camps (coord.), Diálogo e investigación en las aulas (pp. 77-97). Barcelona: Graó.

Barth, B.-M. (2004). L'apprentissage de l'abstraction. París:

Retz.

Bereiter, C., y Scardamalia, M. (1983). Does learning to write have to be so difficult? En A. Freedman; I. Pringle y J. Yalden (eds.), Learning to Write: First Language / Second Language (pp. 20-33). Londres: Longman.

Calil, E., y Myhill, D. (2020). Dialogue, erasure and spontaneous comments during textual composition: What students' metalinguistic talk reveals about newly-literate writers' understanding of revision. Linguistics and Education, 60. doi.org/10.1016/j.linged.2020.100875

Camps, A. (2000). Aprendre gramàtica. En A. Camps y M. Ferrer (coords), Gramàtica a l'aula (pp.101-118). Barcelona: Graó.

Camps, A. (comp.) (2003). Seqüències didàctiques per aprendre a escriure. Barcelona: Graó.

Camps, A. (2017). Escribir es participar en una sociedad alfabetizada. Textos de Didáctica de la Lengua y la Literatura, 75, 3239.

Camps, A., y Fontich, X. (2020). Introduction. En A. Camps y $\mathrm{X}$. Fontich (eds.), Research and teaching at the intersection. Navigating the territory of grammar and writing in the context of metalinguistic activity (pp. 29-43). Bruxelles: Peter Lang. 
Camps, A., y Milian, M. (1999). Introduction. En A. Camps y M. Milian (eds.), Metalinguistic Activity in Learning to Write (pp. 128). Amsterdam: Amsterdam University Press.

Camps, A., Milian, M., Guasch, O., Pérez, F., Ribas, T., y Castelló, M. (2001). Los conceptos gramaticales de los alumnos de secundaria: El pronombre personal. En A. Camps (ed.), El aula como espacio de investigación y reflexión (pp. 161-180). Barcelona: Graó.

Camps, A., y Ribas, T. (coords.) (2017). El verbo y su enseñanza. Hacia un modelo de la enseñanza de la gramática basado en la actividad reflexiva. Barcelona: Octaedro.

Casas, M. (2014). Interacción, uso lingüístico y construcción del saber gramatical en la educación primaria. Tejuelo: Didáctica de la Lengua y la Literatura. Monográfico nº 10, 65-83.

Dolz, J., y Gagnon, R. (dir.) (2018). Former à enseigner la production écrite. Villeneuve d'Ascq, France: Presses Universitaires du Septentrion.

Durán, C. (2010). Parlem de l'adverbi. Els conceptes gramaticals dels alumnes de l'ESO. Articles de Didàctica de la Llengua i la Literatura, 52, 91-11.

Durán, C. (2020). Secondary school students' grammatical representations and metalinguistic activity: The "verb" category. En A. Camps y X. Fontich (eds.), Research and teaching at the intersection (pp. 287-308). Bruxelles: Peter Lang.

Dyson, A. H. (2009). Writing in childhood worlds. En R. Beard, D. Myhill, J. Riley y M. Nystrand (Eds.), The SAGE Handbook of Writing Development (pp. 232-245). London: SAGE.

Flower, L., y Hayes, J.R. (1981). A cognitive process theory of writing. College Composition and Communication, 32(4), 365-387.

Fontich, X., y García-Folgado, M.J. (2018). Grammar instruction in the Hispanic area: The case of Spain with attention to empirical studies on metalinguistic activity. L1-Educational Studies in Language and Literature, 18, 1-39. doi.org/10.17239/L1ESLL2018.18.04.02

Gil, M.R. (2011). Reflexionar sobre la llengua per aprendre a escriure. Guix, 371, 23-26. 
Gil, M.R., y Bigas, M. (2009). L’activitat metalingüística durant una tasca de segmentació d'un text en paraules a segon de primària. Articles de Didàctica de la Llengua i la Literatura, 48, 67-77.

Guasch, O., y Ribas, T. (2013). La entrevista en la investigación cualitativa sobre la didáctica de la lengua. Cultura y Educación, 25(4), 483-488.

Hassan, R. (2004). Analyser des entretiens métagraphiques avec des enfants. Les cahiers THÉODILE, 5, 17-32.

Jaffré, J.-P. (2003). Les commentaires métagraphiques. En J.P. Jaffré (dir.), Faits de langues, dynamiques de l'écriture: approches pluridisciplinaires (pp. 67-76). Paris: Éditions Orphys.

Lantolf, J. (2000). Introducing sociocultural theory. En J. Lantolf (ed.), Sociocultural Theory and Second Language Learning (pp.1-26). Oxford: OUP.

Lantolf, J., y Appel, G. (1994). Theorical Framework: An Introduction to Vygotskyan Approaches to Second Language Research. En J. Lantolf y G. Appel (ed.), Vygotskyan Approaches to Second Language Research (pp.1-32). Norwood NJ: Ablex Publishing Corporation.

Lenz, R. (1912). Para qué enseñar gramática. Conferencia dictada en la Universidad de Chile. Recuperado de https://revistas.uchile.cl/index.php/ANUC/article/viewFile/25606/2692 7

Locke, T. (ed.) (2010). Beyond the Grammar Wars. New York: Routledge.

Mann, S. (2011). A Critical Review on Qualitative Interviews in Applied Linguistics. Applied Linguistics, 32 (1), 6-24.

Mercer, N. (2013). Voces y mentes. Barcelona: Paidós.

Milian (2001). Interacción de contextos en la investigación sobre composición escrita. En A. Camps (coord.), El aula como espacio de investigación y reflexión (pp.23-36). Barcelona: Graó.

Milian, M. (2005). Parlar per "fer gramàtica". Articles de Didàctica de la Llengua i la Literatura, 37, 11-30.

Mondada, L. (2001). L'entretien comme événement interactionnel. En M. Grosjean y J.P. Thibaud (dir), L'espace urbain en méthodes (pp.197-223). Marseille: Éditions Parenthèses. 
Myhill, D., y Jones, S.M. (2015). Conceptualizing metalinguistic understanding in writing. Cultura y Educación, 27:4, 839-867. doi.org/10.1080/11356405.2015.1089387

Myhill, D., Jones, S.M., y Wilson, A. (2016). Writing conversations: fostering metalinguistic discussion about writing. Research Papers in Education, 31(1), 23-44. doi.org/10.1080/02671522.2016.1106694

Myhill, D., Watson, A., y Newman, R. (2020). Thinking differently about grammar and metalinguistic understanding in writing. Bellaterra Journal of Teaching \& Learning Language \& Literature, 13 (2) e870. doi.org/10.5565/rev/jt13.870

Palou, J. (2008). L'ensenyament $i$ aprenentatge del català com a primera llengua a l'escola. Creences $i$ actuacions dels mestres amb relació a les activitats de llengua oral a l'etapa primària. Barcelona: IEC.

RAE-ASALE (2019). Glosario de términos gramaticales. Salamanca: RAE-ASALE y USAL.

Ribas, T. (2001). La regulación del proceso de composición escrita en grupo: Análisis de la utilización de las pautas de revisión. En A. Camps (ed.), El aula como espacio de investigación y reflexión (pp. 51-67). Barcelona: Graó.

Ribas, T., Fontich, X., y Guasch, O. (eds.) (2014). Grammar at school. Research on Metalinguistic Activity in Language Education. Bruxelles: Peter Lang.

Ribas Seix, T., Rodríguez-Gonzalo, C., y Durán, C. (2020). El aprendizaje de la escritura de textos de opinión en alumnos de Primaria: análisis de la actividad metalingüística, de los conceptos sobre la escritura y de los productos finales. Indagatio Didactica, 12 (2), 33-54. doi.org/10.34624/id.v12i2.17436

Rodríguez-Gonzalo, C. (2012). La enseñanza de la gramática: las relaciones entre la reflexión y el uso lingüístico. Revista Iberoamericana de Educación, 59, 87-118. doi.org/10.35362/rie590458

Rodríguez-Gonzalo, C., y Durán, C. (2019). Relaciones entre conceptualización y uso en el aprendizaje de la gramática. Dos investigaciones de aula sobre el verbo. En A. Leal; F. Oliveira; F. Silva; I.M. Duarte; J. Veloso; P. Silvano y S. Rodrigues (orgs.), A linguística 
na formação do professor: das teorias às práticas (pp. 181-206). Porto: FLUP. doi: 10.21747/978-989-8969-20-0/linga11.

Salas, N., Birello, M., y Ribas, T. (2020). Impact of an SRSD writing intervention on the spelling and reading comprehension skills of low- and mid-high SES children. Reading and Writing 34, 1653-1680. doi.org/10.1007/s11145-020-10103-8

Talmy, S. (2011). The Interview as Collaborative Achievement: Interaction, Identity and Ideology in a Speech Event. Applied Linguistics, 32 (1), 25-42.

Torralba, M. (2012). La comprensió dels temps verbals a primària. Estudi descriptiu dels coneixements dels alumnes de quart $i$ sisè sobre el verb com a codificador temporal. Tesi doctoral no publicada. Barcelona: UAB.

Vigotski, L.S. (1988). Pensament $i$ llenguatge. Vic: Eumo/Diputació de Barcelona. 
Tejuelo, nº 35.2 (2022), págs. 103-136. La entrevista sobre la escritura con alumnado de Primaria...

136 | Pág in a 